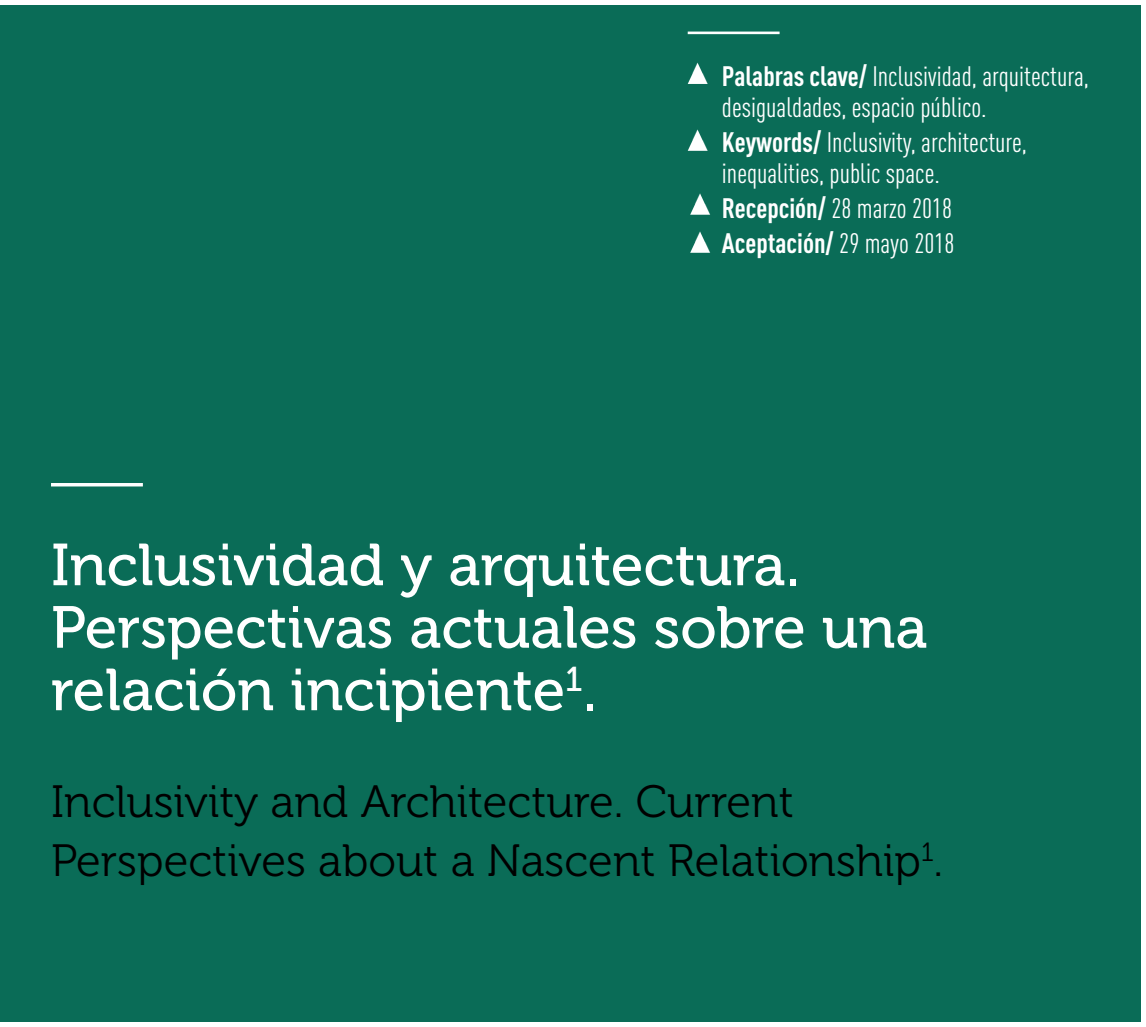

\section{Rebeca Silva-Roquefort}

Diseñadora Industrial, Universidad de Chile Chile.

Diploma de Estudios Avanzados en Urbanística y ordenación del territorio, Universidad Politécnica de Madrid, España

Doctora en Arquitectura y Urbanismo,

Universidad Politécnica de Madrid, España.

Profesora Asistente, Instituto de la Vivienda,

Facultad de Arquitectura y Urbanismo,

Universidad de Chile, Chile.

bekysilva@uchilefau.cl

\section{Mariela Gaete-Reyes}

Arquitecta, Universidad Nacional de Colombia Colombia.

Magíster en Desarrollo Urbano, Pontificia

Universidad Católica de Chile, Chile.

Doctora en Geografía, King's College of London, Inglaterra.

Profesora Asistente, Instituto de la Vivienda

Facultad de Arquitectura y Urbanismo,

Universidad de Chile, Chile.

m.gaete-reyes@uchilefau.cl

\section{Luis Campos-Medina}

Sociólogo, Universidad de Chile, Chile. Máster en Ciencias Sociales, École des Hautes Études en Sciences Sociales (EHESS), Francia.

Doctor en Sociología, École des Hautes Études en Sciences Sociales (EHESS), Francia. Profesor Asistente, Instituto de la Vivienda

Facultad de Arquitectura y Urbanismo,

Universidad de Chile, Chile.

luiscampos@uchilefau.cl

RESUMEN/ El presente artículo busca mostrar el estado del arte de la inclusividad en el ámbito urbano-habitacional chileno, así como sugerir la relevancia que esta tiene para la construcción de ciudades más justas. Para ello, se declara la estrategia metodológica del análisis presentado; se expone la noción "inclusividad' desde los conceptos de accesibilidad a los que tradicionalmente se le asocia; se destacan los avances y problemas desde los dispositivos legales que organizan en Chile la producción del espacio urbano (Ordenanza General de Urbanismo y Construcciones); y, se articula la revisión conceptual y legal para caracterizar y evaluar espacios urbanos desde la perspectiva de la inclusividad. Se concluye con una propuesta sobre cómo la arquitectura debería abordar de manera inclusiva las distintas formas de exclusión actuales para el desarrollo de ciudades más justas y amigables. ABSTRACT/ This article aims at revealing the state of the art of inclusivity in the Chilean urban-housing sphere, as well as suggesting its relevance for the construction of fairer cities. For such purpose, the methodological strategy of the analysis presented is stated; the notion of "inclusivity" is explained using the concepts of accessibility to which it has been traditionally associated; progresses and issues from the legal devices that arrange the production of the urban space in Chile (General Ordinance of Urban Development and Construction) are highlighted; and the conceptual and legal revision is articulated to characterize and assess urban spaces from a perspective of inclusivity. The work concludes with a proposal regarding how architecture should inclusively address the different forms of current exclusions for the development of fairer and friendlier cities.

\section{INTRODUCCIÓN.}

contemporáneas es posible observar una serie de barreras -tanto materiales como inmateriales- que generan dificultades de acceso a espacios y recursos (Cordero 2009). En su asentamiento y permanencia, tales barreras se convierten en formas de exclusión para diversos grupos de la sociedad. Enfrentar esas formas de exclusión y modificarlas es una preocupación que ha tomado fuerza y relevancia pública en los últimos años, siendo una de las perspectivas conceptuales más desarrolladas, justamente, la que se interroga sobre la inclusión y, en particular

\begin{abstract}
Este texto abordará la manera en que la arquitectura, en tanto disciplina encargada de la producción material del espacio urbano, ha recogido el desafío de afrontar las barreras antes indicadas o, dicho en otras palabras, la manera en que se ha hecho cargo de la problemática de la inclusividad. Para ello, en primer lugar, se explicita la estrategia metodológica seguida de modo de esclarecer el tenor y validez del análisis presentado. En segundo lugar, se examina la perspectiva conceptual trabajada, indicando las características del tránsito desde la noción de inclusión a la inclusividad y explicitando el modo específico en el que se entiende esta última
\end{abstract}

noción. En tercer lugar, se aborda la forma en la que se ha considerado la problemática de la inclusividad en el principal instrumento legal que organiza la producción del espacio urbano -la Ordenanza General de Urbanismo y Construcciones (OGUC)-, destacando los principales avances que se han producido a lo largo del tiempo y las principales problemáticas. En cuarto lugar, se analiza la manera en que se ha materializado la normativa en tres casos de estudio concretos. El artículo finaliza con un apartado de conclusiones donde se ofrece una propuesta respecto del modo en que la arquitectura debería recoger la problemática de la inclusividad y la relevancia social que esto tendría. 


\section{PANORÁMICA CONCEPTUAL DE LA INCLUSIVIDAD. \\ De la inclusión a la inclusividad.}

La inclusión tiene una larga historia en la reflexión filosófica y política (Robles 2005). El concepto se sitúa en la discusión acerca del perfeccionamiento democrático y, en consecuencia, tiene su raíz en el pensamiento filosófico liberal. Con él se busca indicar que las sociedades contemporáneas generan procesos de exclusión derivados de la desigual distribución de recursos (Savidan 2015; Tilly 2000) y del operar de los mecanismos productivos y distributivos del régimen capitalista y que, frente a ellos, la sociedad democrática debe desarrollar dispositivos

METODOLOGÍA. La metodología aplicada contempló tres momentos. Primero, se efectuó una revisión exhaustiva de bibliografía pertinente, concentrada en trazar la problemática conceptual de la inclusión y, especialmente, en la elaboración de una propuesta propia del concepto de inclusividad. A esto se agregó el análisis de las 15 publicaciones nacionales periódicas más relevantes en el ámbito de la arquitectura y el urbanismo (tabla 1). En seguida, se efectuó una sistematización y análisis de contenido de la Ley N²0.422, poniendo especial énfasis a la OGUC y al Decreto N 50 . Finalmente, se elaboró un instrumento de observación y análisis en terreno, articulando elementos conceptuales y legales. Este instrumento fue denominado 'ficha de evaluación inclusiva' y con él se hizo posible desarrollar un levantamiento planimétrico, un registro fotográfico y un análisis de las condiciones espaciales de tres casos de estudio seleccionados. El levantamiento de información de los tres casos se realizó durante el segundo semestre de 2017. que eviten el surgimiento y la consolidación de grupos marginados (Beck, Lash y Giddens 1997; Giddens 1996; Habermas 1989). La inclusión busca, así, garantizar la libertad de los individuos, reconociendo su diversidad, y poniéndolos a todos bajo un marco institucional común, en el que se "garanticen sus derechos de libertad e igualdad y se les pueda exigir responsabilidad por sus actos" (Bilbeny 1999: 113). El problema con esta idea de inclusión es que esa instalación bajo un mismo marco común se ha hecho, generalmente, de acuerdo a procedimientos de agregación o de asimilación. Los primeros consisten en sumar grupos diferentes, pero sin preocuparse por su integración efectiva, lo que facilita el surgimiento de formas de segregación. Los segundos promueven la fusión de diferencias, borrándolas en un marco común.

Frente a esto, se ha propuesto la necesidad de avanzar en la comprensión de la inclusión como "integración pluralista" (Bilbeny

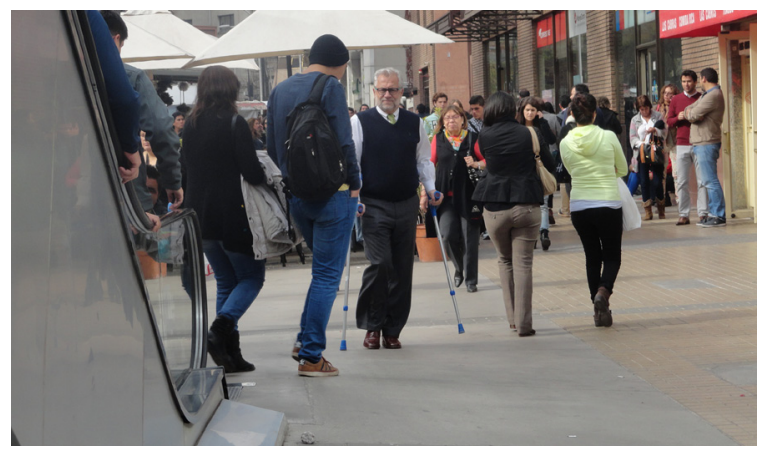

Imagen 1. El reconocimiento de la diversidad como enfoque inclusivo (fuente: Rebeca Silva).

1999: 114), la que se caracteriza ya no por proponer una sumatoria o combinación de diversidades, sino por la producción conjunta de una colectividad nueva. La idea de inclusividad que proponemos en este texto se asienta en esta idea: generar inclusión a través de la integración pluralista de la diversidad, lo que implica que se debe propender a un reconocimiento legal de la diversidad y avanzar hacia un respeto social de la misma (Bilbeny 1999).

Desde esta perspectiva, instalar la interrogante por la inclusividad en el ámbito urbano-habitacional cobra relevancia, puesto que las ciudades son -y han sido históricamente- el espacio de aparición y surgimiento de la diversidad social y la desigualdad (Joseph 1994), pero, al mismo tiempo, han sido el espacio de producción de las herramientas para enfrentarlas y para generar nuevas formas de ciudadanía, más profundas y adecuadas a las exigencias de los tiempos.

Preguntarse por la inclusividad en el espacio urbano-habitacional es, en consecuencia, preguntarse por las condiciones reales y 
visibles -es decir, posibles de ser sometidas al escrutinio público- existentes para apoyar la realización de sujetos diversos. Esto equivale a decir que los individuos y grupos requieren de apoyos e infraestructuras tanto sociales como materiales para poder ejercer su libertad (Butler 2017) y conseguir sus objetivos, y que es parte del pacto democrático generar, de modo colectivo, condiciones para ello. La producción arquitectónica del espacio urbano es, en consecuencia, un ámbito real y concreto en el que se plasma la pregunta por la inclusividad. De esta forma, la inclusividad en la arquitectura puede ser definida como la generación de infraestructuras y de dispositivos materiales para dar acceso al espacio urbano y construido, con el propósito de hacer posible el despliegue de las capacidades, el disfrute y la realización de los sujetos que componen la sociedad, todo ello con base en el reconocimiento activo y permanente de la desigualdad y la discriminación

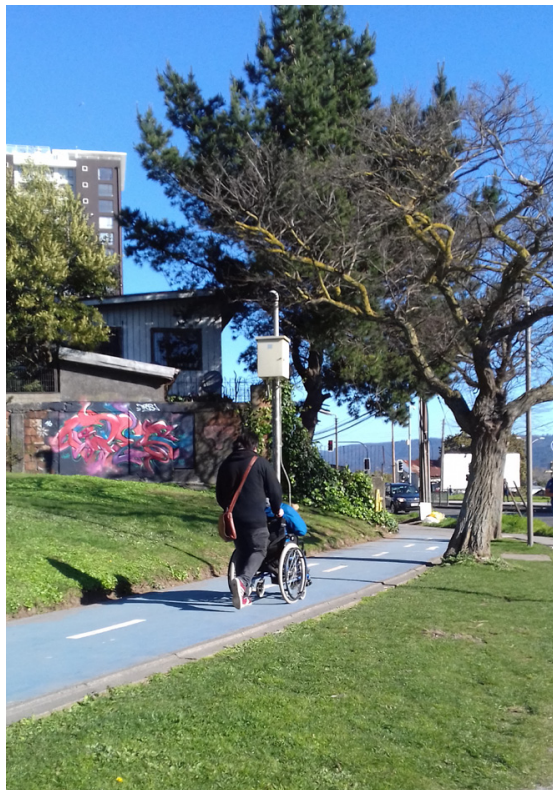

Imagen 2. Cómo aparecer o desaparecer en la ciudad (fuente: Los autores 2017)
Presencia de la inclusividad en la discusión arquitectónica chilena.

A pesar de la relevancia que trasunta una definición como la recién propuesta, la revisión de una gran cantidad de artículos publicados en revistas de corriente principal pone en evidencia la existencia de un vacío en cuanto a una visión integral del concepto de inclusividad. En efecto, en la discusión disciplinar se produce una suerte de asimilación del concepto de inclusividad al de accesibilidad, restringiendo su espectro de operación al plano físico y material. Dicho de otra forma, lo que pone en evidencia la revisión realizada es que el concepto, como tal, casi no aparece y que lo que aparece en relación a los conceptos emparentados, como inclusión, exclusión, integración o accesibilidad, es e par inclusión-accesibilidad (De la Fuente y Hernández 2013; Lotito y Sanhueza 2011;). Para demostrarlo, en la Tabla 1 se presenta la sistematización de los hallazgos encontrados en quince publicaciones periódicas chilenas ${ }^{2}$.

\section{MODIFICACIONES LEGALES: Ley $\mathbf{N}^{\circ} 20.422$, Decreto $\mathbf{N}^{\circ} 50$ y cambios a la OGUC. En la historia chilena reciente, el} año 2010 marca un hito en la perspectiva de la inclusividad. El 10 de febrero de ese año fue publicada en el Diario Oficial la Ley $N^{\circ} 20.422$, que establece normas sobre igualdad de oportunidades e inclusión social de personas con discapacidad, y cuyo objetivo es "asegurar el derecho a la igualdad de oportunidades de las personas con discapacidad, con el fin de obtener su plena inclusión social, asegurando el disfrute de sus derechos y eliminando cualquier forma de discriminación fundada en la discapacidad" (Ministerio de Planificación, Gobierno de Chile 2010, artículo ${ }^{\circ}$ ). Esta ley vino a reemplazar a la Ley $N^{\circ} 19.284$, promulgada en 1994, que establecía las normas para la plena integración social de personas con discapacidad.

La Ley N²0.422 se organiza a partir de una serie de principios (artículo $3^{\circ}$ ), entre los que cuentan: la intersectorialidad, la vida independiente, la accesibilidad universal,

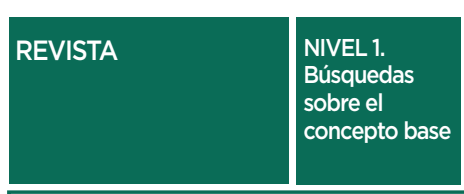

NIVEL 2.
Búsquedas
sobre
conceptos
asociados

NIVEL 3.
Búsquedas
con otras
articulaciones
del concepto

NIVEL 4.

Otras variantes

en menor

grado de

conexión

\begin{tabular}{|l|l|}
\hline 1. Auca \\
\hline 2. CA \\
\hline 3. ARQ \\
\hline 4. Revista de Arquitectura \\
\hline 5. Revista de Urbanismo \\
\hline 6. Revista INVI \\
\hline 7. Revista Eure \\
\hline 8. ARS \\
\hline 9. Arquitecturas del sur \\
\hline 10. Cuadernos de \\
arquitectura \\
\hline 11. AUS \\
\hline 12. AOA \\
\hline 13. Revista 180 \\
\hline 14. Márgenes \\
\hline 15. Materia \\
\hline
\end{tabular}

Tabla 1. Resultado de búsqueda en revistas chilenas (fuente: Elaboración propia) 
el diseño universal y la participación, y el diálogo social. No obstante, la importancia de todos estos principios, para efectos de nuestra argumentación nos remitiremos al principio de intersectorialidad. Esto se justifica porque tal principio indica que lo que se pretende desde el aparato estatal, es que las políticas de cualquier ámbito de la gestión pública consideren los derechos de las personas con discapacidad como elementos transversales. Atendiendo a esta condición es que surge el Decreto N 50 que modifica la OGUC y que establece que, a partir de su fecha de publicación (4 de marzo de 2016) se vuelve exigible una serie de requisitos de accesibilidad para las construcciones nuevas (Ministerio de Vivienda y Urbanismo, Gobierno de Chile 2016). Este Decreto sintetiza un conjunto de transformaciones que pueden ser organizadas en cinco tipos de cambios en:

- Los vocablos y definiciones: por ejemplo, accesibilidad universal, huella podotáctil, ruta accesible, entre otros.

- Los estándares: por ejemplo, los estándares de veredas (art. 2.2.8.), las medidas de estacionamientos (art. 2.4.2), entre otros.

- Las facultades y requisitos: destaca la incorporación del Plano y Memoria de Accesibilidad (art. 5.1.6 numeral 14). Además, se modifica la idea de que solo debía haber accesos especiales para personas con discapacidad, incorporándose que debe existir rutas accesibles pensadas y diseñadas para esas personas.

- Los ámbitos de aplicación: destaca la incorporación dentro de los alcances de esta ley no solo de los edificios nuevos, sino también de los existentes. Es posible agrupar estas exigencias en dos períodos, los referidos a "ajustes necesarios" y los referidos a "exigencias de accesibilidad".

\section{- El instrumental técnico para llevar a} efecto la ley: la inclusión de la noción de ruta accesible genera la necesidad de su representación gráfica y su explicación narrativa, lo que redunda en el requerimiento de elaborar dos instrumentos nuevos, como son el plano y la memoria de accesibilidad, a cuya explicación se aboca el Documento Técnico de Referencia (DTR) Accesibilidad 2016/001010
Aunque las transformaciones legales remiten a la discapacidad y la accesibilidad -fundamentalmente de "rampas y barreras" (SENADIS, CECH y UCSH 2010)-, dan cuenta de transformaciones en la línea de un mayor reconocimiento de la diversidad y la desigualdad, y de una ampliación del repertorio de herramientas para enfrentarlas. La OGUC, por tratarse de un documento normativo y de aplicación en la construcción, debe transformar la accesibilidad en un conjunto de modificaciones materiales, posibles de ser exigidas, revisadas y corroboradas en las construcciones. Por ello, se vuelve relevante observar el modo en que la ley se plasma,

materialmente, en el espacio construido

CASOS DE ESTUDIO. De acuerdo

al procedimiento previsto, luego de la revisión conceptual y de la sistematización legal, se pasó a elaborar una 'ficha de evaluación inclusiva', instrumento que buscó caracterizar cada uno de los casos seleccionados en materia de inclusividad. La ficha se basó en la metodología de evaluación ergourbana (Silva 2017) y se orientó de acuerdo a los criterios propuestos por la OGUC. La ficha se compone de cuatro partes: la primera es la presentación del caso, con el resumen de los aspectos analizados en él; la segunda identifica las situaciones críticas derivadas de la falta de equipamiento o de las malas condiciones en que se encuentra; en la tercera parte se abordan las interacciones sociales y dinámicas identificadas al observar el uso del espacio; y, en la cuarta y última, se chequea punto a punto el cumplimiento de lo establecido en las tablas resumen de la normativa de accesibilidad. La ficha se aplicó a tres casos de estudio: - El primer caso fue la remodelación del Parque Quinta Normal, identificado en la revista AOA (2014) como una obra con características de accesibilidad reconocibles en proyecto de mejoramiento urbano del espacio público de Santiago. Su relevancia se debe a que es un espacio público de importancia histórica en la ciudad de Santiago y responde a un programa que busca rehabilitar el parque y fomentar su uso.

- El segundo caso seleccionado fue la Villa Portales, identificado en la revista AUCA (1967). Su importancia se debe a su condición de conjunto habitacional de envergadura, de relevancia arquitectónica en tanto obra vanguardista, destacada por generar espacios urbanos intermedios como espacios de integración para todos los habitantes. Este proyecto consideró, específicamente, rampas de acceso al edificio, de manera de permitir a personas con movilidad reducida ingresar por una vía alternativa a la escalera.

- El tercer caso escogido fue el Centro Cultural Espacio Matta, detectado en la revista AOA (2014), el cual destaca principalmente porque el proyecto incorpora elementos requeridos por la normativa de accesibilidad universal (rampas, baños accesibles, ascensor, entre otros). El caso fue seleccionado para identificar cómo la normativa se refleja en un proyecto de arquitectura actual que, además, en su condición de centro cultural, es un lugar de encuentro

\section{heterogéneo y dinamico.}

\section{Aplicación de la ficha de evaluación} inclusiva ${ }^{3}$.

constatar que la intervención inclusiva es insuficiente y no responde a las necesidades de los usuarios reales. Asimismo, el equipamiento 'inclusivo' existente no está integrado en sus diversos componentes de manera sistémica. Se observa que las soluciones propuestas son de tipo estándar y, muchas veces, no satisfacen las prácticas de aquellos usuarios sin problemas de accesibilidad (imagen 3).

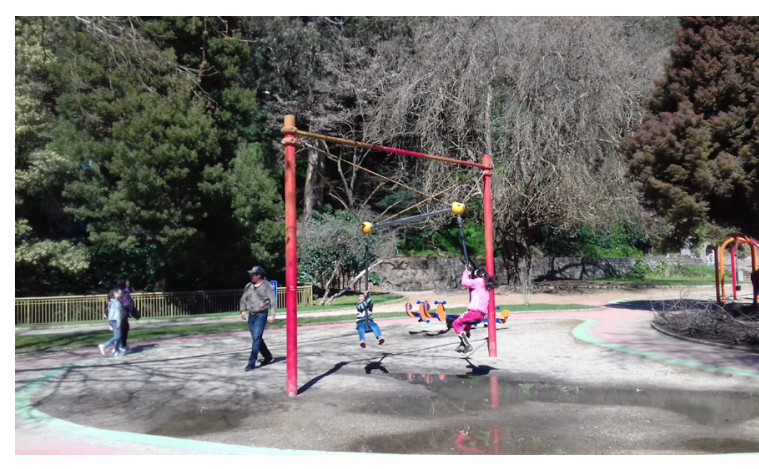

Imagen 3. Ejemplo de la incomprensión del contexto y los usos reales del equipamiento (fuente: Los autores 2017). 


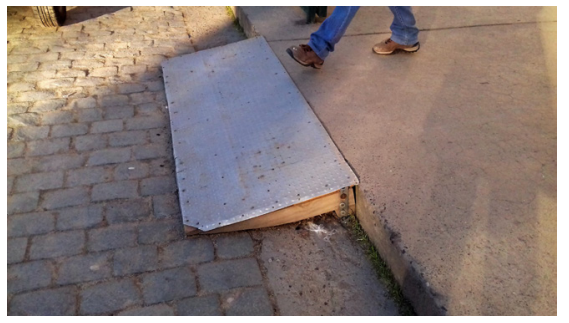

Imagen 4. Quinta Normal. La accesibilidad resuelta mediante soluciones que no abordan el problema de fondo (fuente: Los autores 2017).

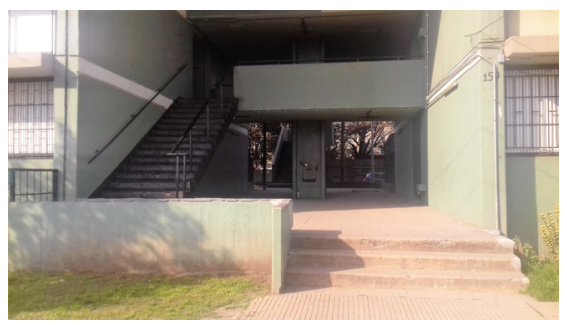

Imagen 5. Villa Portales. La ausencia de rampas y las malas miten la accesibilidad a la vivienda (fuente: Los autores 2017).

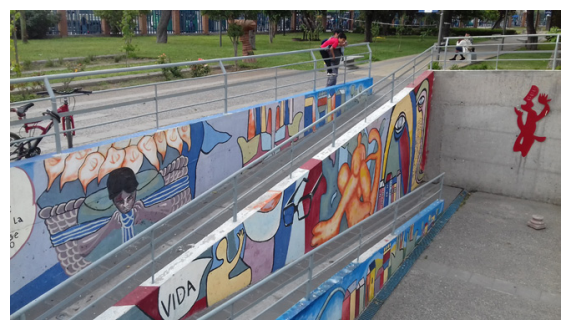

Imagen 6. Espacio Matta. Rampas de acceso que cumplen con barandas, pero tienen pendientes elevadas y descansos insuficientes (fuente: Los autores 2017).
Los resultados específicos indican que en Villa Portales y en el Parque Quinta Normal existen serios problemas de accesibilidad. Estos espacios son emblemáticos de la ciudad de Santiago, por lo que parece crucial que cuenten con elementos mínimos de accesibilidad, como rampas o pasamanos en todos sus accesos, lo cual no sucede. Ambos se encuentran en claro deterioro, a pesar de las obras de remodelación que se han llevado a cabo en cada uno.

En el caso de la remodelación del Parque Quinta Normal, uno de los principales problemas de accesibilidad refiere a la materialidad de las superficies del piso, que es inapropiada, ya que se utiliza adoquín (tipificado como material no accesible). Además, se observa un deterioro de las superficies, el que se transforma recurrentemente en una barrera que impide el libre desplazamiento de las personas. Por último, se constata la presencia de soluciones superficiales espacio para generar accesos con rampas y otros materiales que quedan desconectados de programa global del espacio (imagen 4). En el caso de Villa Portales, existen serios problemas que hacen que el espacio sea poco accesible e inclusivo. Las superficies presentan deterioro, hay ausencia de rampas y ascensores, los cruces y veredas se encuentran en malas condiciones y no existe una clara conectividad entre los diferentes espacios (imagen 5).

El espacio con mayor presencia de elementos de accesibilidad es el Centro Cultural Espacio Matta, en el que se observa un programa con intención de dar mayor acceso a las personas. Sin embargo, también se confirman deficiencias, tales como rampas de acceso sin descansos adecuados, ausencia de barandas en otras rampas (también de acceso), falta de textura en la huella exterior que permita distinguir el inicio y final del recorrido, inexistencia de huella podo-táctil al interior del centro (existe una sola huella en la calle), y la altura estándar del mesón de atención, que no permite el acercamiento de personas en silla de ruedas o de niños (imagen 6)

La información recopilada permitió reconocer que, en general, las intervenciones con carácter 'inclusivo' carecen de equipamientos e infraestructura accesible y que, cuando tales equipamientos existen, carecen de una integración sistémica que permita dar continuidad y legibilidad al uso del espacio. Del análisis de la información generada en las fichas, surgen algunas problemáticas recurrentes que pueden ser tipificadas del siguiente modo:

- Falta de claridad en el modo de usar y acceder al espacio.

- Falta de continuidad en la ruta de acceso y desplazamiento.

- Deterioro progresivo de infraestructuras, superficies y/o equipamientos.

- Presencia de soluciones coyunturales y paliativas al uso del espacio ('soluciones parche')

- Falta de integración sistémica de las soluciones inclusivas de uso del espacio.

- Descontextualización en la implementación de los lugares. Se aplican soluciones sin considerar las características propias de cada lugar. 
CONCLUSIONES. El trabajo de revisión bibliográfica desarrollado indica que el tratamiento de la inclusividad, desde el diseño arquitectónico es, a la vez, restringido e incipiente. Restringido, porque la inclusividad tiende a ser limitada a la accesibilidad y al tratamiento de los problemas de discapacidad y movilidad reducida. Incipiente, porque, no obstante contar con tradiciones conceptuales que ya tienen décadas de existencia (por ejemplo, el diseño universal), no se ha profundizado en las problemáticas de accesibilidad recién indicadas, ni tampoco en la articulación de las problemáticas de accesibilidad con otras que no tienen una manifestación físico-espacial tan evidente, pero que son igualmente modeladoras de la experiencia de ciudad y de la calidad de vida. Si, como dijimos al principio, nuestras ciudades son el escenario privilegiado para la expresión, tanto de las desigualdades como para la generación de la acción concertada tendiente a su reducción, resulta fundamental comenzar a proyectar ciudades considerando la diversidad social existente y las diferencias de experiencia -y disfrute- asociadas a ellos. En este sentido, consideramos que el diseño que se hace cargo de la inclusividad reconoce la interacción dinámica entre el medio construido, el individuo y su comunidad, y entiende que esa es una interacción permanente, cambiante y multiforme, en la que inciden las definiciones disciplinares, la legalidad y la práctica concreta.

Para finalizar, cabe hacer explícito que el enfoque de inclusividad tiene una fuerte vocación transformadora y una clara impronta ética, puesto que busca generar ciudades más justas y más vivibles para todas las personas. En ese sentido, el enfoque de inclusividad no puede quedar restringido a variables de orden legal, sino que debe plasmarse en el espacio urbano existente con enfoque de derecho. La noción de inclusividad que hemos propuesto busca mostrar que la arquitectura y el urbanismo tienen mucho que decir sobre las posibilidades de aparición en el espacio público urbano y sobre la participación en la vida en común en una sociedad democrática. Apelamos a avanzar en una retroalimentación virtuosa, que comience con intervenciones en el espacio construido de la ciudad, y que, a su vez, se conviertan en espacios para la participación y el ejercicio de la ciudadanía, contribuyendo a inspirar marcos legales y de reconocimiento más inclusivos. La arquitectura puede y debe contribuir a ello. $\mathbf{\Delta}$

\section{REFERENCIAS}

Beck, U., Lash, S. y Giddens, A., 1997. Modernización reflexiva. Politica tradición y estética en el orden social moderno. España: Alianza Editoria!

Bilbeny, N., 1999. Democracia para la diversidad. Barcelona: Editorial Ariel.

Butler, J., 2017. Cuerpos aliados y lucha politica. Buenos Aires: Editorial Paidós.

Cordero, E., 2009. "Editorial." Revista AUS, 6, 5.

De la Fuente, Y. y Hernández, J., 2013. "Rehabilitación de viviendas con criterios de accesibilidad en España Respuestas inclusivas ante la diversidad humana y social." Revista de Urbanismo, 28, 60-78 Giddens, A., 1996. Más allá de la izquierda y la derecha. El futuro de las politicas radicales. España: Editorial Cátedra.

Habermas, J., 1989. Problemas de legitimación en el capitalismo tardio. Madrid: Editorial Amorrortu. Joseph, I., 1994. El transeúnte y el espacio público. Barcelona: Editorial Gedisa.

Lotito, F. y Sanhueza, H., 2011. "Discapacidad y barreras arquitectónicas: Un desafio para la inclusión." Revista AUS, 9, 10-13.

Ministerio de Planificación, Gobierno de Chile, 2010. Ley No20.422 que establece normas sobre igualdad de oportunidades e inclusión social de personas con discapacidad

Disponible en: https://www.leychile.cl/Navegar?idNorma=1010903
Ministerio de Vivienda y Urbanismo, Gobierno de Chile, 2016. Decreto N50 que modifica Decreto Supremo №47, de vivienda y urbanismo, de 1992, ordenanza general de urbanismo y construcciones en el sentido de actualizar sus normas a las disposiciones de la Ley $N^{0} 20.422$, sobre igualdad de oportunidades e inclusión social de personas con discapacidad. Disponible en: https://www.leychile.cl/Navegar?idNorma=1088117 Robles, F., 2005. "Contramodernidad y desigualdad social. Individualización e individuación, inclusión/ exclusión y construcción de identidad. La necesidad de una sociologia de la exclusión." Revista MAD, 12, 1-31. Savidan, P., 2015. Voulons-nous vraiment l'égalité. Paris: Albin Michel.

Servicio Nacional de la Discapacidad (SENADIS), Conferencia Episcopal de Chile (CECH) y Universidad Católica Silva Henriquez (UCSH), 2010. Rampas y Barreras: Hacia una cultura de la inclusión de las personas con discapacidad. Santiago de Chile: Andros

Silva, R., 2017. "Ergociudad. Mirar la ciudad, lugar de residencia de las personas." En Imilan, W., Larenas, J., Carrasco, G. y Rivera. S. (Eds.), ¿Hacia dónde va la vivienda en Chile? Nuevos desafios en el hábitat residencial Santiago de Chile: Adrede Editora.

Tilly, C., 2000. La desigualdad persistente. Buenos Aires: Editorial Manantial. 